\title{
Marheineke, Schleiermacher und das Reformationsjubiläum
} von 1817

$\ln :$

Reformation und Moderne : Pluralität - Subjektivität - Kritik ; Akten des Internationalen Kongresses der Schleiermacher-Gesellschaft in Halle (Saale), März 2017 / herausgegeben von Jörg Dieken, Arnulf von Scheliha und Sarah Schmidt. - Berlin: De Gruyter, 2018 ISBN: 978-3-11-056766-3

(Schleiermacher Archiv ; 27)

S. $169-180$ 


\title{
Marheineke, Schleiermacher und das Reformationsjubiläum von 1817
}

\section{Das Jubiläum von 1817}

\begin{abstract}
Marheineke's Reformationsgeschichte [...] gefällt mir beim ordentlichen Lesen weit weniger als beim ersten Blättern. Es ist doch gar zu wenig eigentliche Composition darin, und in den Auszügen wiederum zu viel fremdartiges mit aufgenommen. Das politische und literarische ist fast ganz vernachlässigt; und im Stil ist auch der gute Vorsaz sich dem volksthümlichen anzunähern auf der einen Seite ins abenteuerliche hineingetrieben, auf der andern nichts weniger als treu gehalten. ${ }^{1}$
\end{abstract}

So schrieb Friedrich Schleiermacher am 15. September 1817 an seinen Freund, den Hallenser Philologen und reformierten Prediger Gottfried Ludwig Blanc. Schleiermacher und der zwölf Jahre jüngere Marheineke waren Kollegen an der Berliner theologischen Fakultät, später sollten sie es auch als Pfarrer an der Dreifaltigkeitskirche werden, und beide beteiligten sich am 300jährigen Jubiläum des Lutherschen Thesenanschlags. Schleiermacher sollte bei der akademischen Feier am dritten November die lateinische Festrede halten und hatte zur Vorbereitung darauf Marheinekes schon vorliegenden Hauptbeitrag zum Jubiläum studiert, die Geschichte der teutschen Reformation. Kontakt zwischen beiden hatte nicht erst bestanden, seit Schleiermacher Marheineke Ende 1810 nach Berlin berufen hatte, ${ }^{2}$ sondern schon seit 1806: Marheineke schrieb Schleiermacher, dessen Reden über die Religion hätten in ihm eine wohltätige Veränderung gezeitigt, er hat seinerseits aber mit seinem Konzept einer Universalkirchenhistorie, die die Objektivierung der idealen Religion in der Geschichte beschreibt, wohl auch auf Schleiermacher gewirkt. ${ }^{3}$

Das Jubiläum wurde in ganz Deutschland im großen Stil begangen, selbst in den katholischen Bundesstaaten. Die letzten Jahre hatten die Befreiung vom Joch Napoleons und eine politische Neuordnung gebracht; das Jubiläum wurde Anlass zur Selbstdeutung, Selbstfindung und Bestandsaufnahme des Protestantismus, die denn auch sehr mannigfaltig geriet. Man feierte den Ausbruch aus Unwissenheit und Unmündigkeit, den Sieg von Vernunft, Toleranz und Selbstbestimmung über die Unterdrückung der Geister und Gewissen, verglich aber auch andererseits die seinerzeitige päpstliche Hegemonie mit dem nicht weniger tyrannischen Rationalismus der Gegenwart. Luthers deutsche Befreiungstat schlug die Brücke zu Marschall Vorwärts und

1 Wilhelm Dilthey (Hg.), Aus Schleiermacher’s Leben. In Briefen, Bd. 4, Berlin 1863, 222.

2 Friedrich Schleiermacher, Brief *3480, KGA V/11, hg.v. Simon Gerber / Sarah Schmidt, Berlin / New York 2016, 456.

3 Vgl. Simon Gerber, Schleiermachers Kirchengeschichte, Tübingen 2015, 91-95.

https://doi.org/10.1515/9783110569520-012 
anderen Helden des beendeten Krieges, seine Bibelübersetzung mahnte zu neuer Sammlung um das göttliche Wort, sein Bekennermut hielt der schlaffen, glaubensarmen Gegenwart den Spiegel vor. ${ }^{4}$ In gewisser Weise erkennt man darin schon das 500. Jubiläum der Reformation: Laut der Sonderausgabe des evangelischen Magazins chrismon zum 31. Oktober 2016 ist Reformation ein Aufruf zu Engagement und konkretem Handeln, sie bedeutet, für Gerechtigkeit, Demokratie und Freiheit einzutreten, immer auf der Suche zu bleiben, für etwas zu kämpfen, statt sich mit Dingen bloß abzufinden, aber auch, in der Politik sich auf vorletzte Dinge zu beschränken und in Anbetracht der Freiheit eines Christenmenschen nicht dem Perfektionismus und anderen Ideologien $\mathrm{zu}$ verfallen. ${ }^{5}$

\section{Marheinekes Beiträge zum Jubiläum}

Marheineke veröffentlichte 1818 eine Sammlung mit u. a. fünf Reformationspredigten, davon zweien vom Jubiläumsjahr 1817. Von diesen zwei hat er offenbar nur eine tatsächlich gehalten, nämlich nach Angabe des Buches am 14. September in Hanau, der Heimat seiner Frau, während die andere Predigt eine reine Lesepredigt war. Die Hanauer Predigt hat die Geschichte von Jesu Sturmstillung zum Text (Mt 8,23-28): Zu den Stürmen, die das Schifflein Christi bis heute erschütterten, gehöre die Verpflanzung westeuropäischer Flachheit mit ihrem oberflächlichen Witz und ekelhaften Leichtsinn auf den Boden der deutschen evangelischen Kirche; all das sei nicht den Reformatoren anzulasten, wie denn auch der Sturmwind nicht von Christus oder seinem Schiff herkomme, sondern immer von außen. Wind und Wellen sei es aber zugleich zu verdanken, dass das Schiff stets weiterfahre und sich die Besatzung mit Christi Hilfe im Glauben bewähre, und so sei die Kirche nunmehr auch schon nicht mehr da, wo sie zur Zeit der Reformation gewesen sei. ${ }^{6}$ Die Lesepredigt - sie geht über Jesu Wort von der Wahrheit, die frei macht (Joh 8,31-32) - sieht das Göttliche der

4 Vgl. Theologische Nachrichten 1818, 37-43. 66-87.122-165.197-245.263-293.321-337.367-393.427433 (Berichte über die staatlichen und kirchlichen Bestimmungen und über die Feiern in den deutschen Staaten, Lieder und Texte zum Jubiläum); Lutz Winckler, Martin Luther als Bürger und Patriot, Lübeck / Hamburg 1969; Wichmann von Meding, Kirchenverbesserung, Bielefeld 1986; Dorothea Wendebourg, „Die Reformationsjubiläen des 19. Jahrhunderts“, ZThK 108 (2011), 270 - 335, hier 278279.284-285.290-298.311-319.

5 „Was mir Reformation bedeutet“, chrismon spezial, das evangelische Magazin zum Reformationstag 2016, 4- 5. - Vgl. auch Wichmann von Meding, „Die Reformation bejubeln? Ein ungehaltener Vortrag“, DtPfrBl 116 (2016), 492-496, der einen kritischen Durchgang durch verschiedene Versuche, die Reformation und ihre Gegenwartsbedeutung zu würdigen, macht.

6 Philipp Marheineke, Fünf Reformations-Predigten nebst mehreren andern Religionsvorträgen, Berlin 1818, 25-54, wieder abgedruckt in: Sammlung auserlesener Jubel-Predigten und Gedichte. Zur Erinnerung an das dritte Jubel-Fest der evangelischen Kirche, hg. v. Valentin Carl Veillodter / Christian Schreiber, Allgemeine Chronik der dritten Jubel-Feier der deutschen evangelischen Kirche 2,1, Erfurt und Gotha 1819, 6-14. 
Reformation darin, dass Christus sich der ihm entfremdeten Welt aufs Neue geschenkt habe, dass der durch klerikale Übergriffe getrübte christliche Staat wiederhergestellt worden sei und dass die übersinnliche Wahrheit des Glaubens sich nun immer wieder neu in zeitgemäßen Formen des Kultus und der Kirchenverfassung darstellen könne, wozu denn auch die Union der Lutheraner und Reformierten gehöre. ${ }^{7}$

Marheinekes Hauptbeitrag zum Jubiläum ist aber, wie gesagt, seine zweibändige Geschichte der teutschen Reformation. Neuere gelehrte Darstellungen der Reformationsgeschichte gab es damals, etwa im Rahmen der mehrbändigen Kirchengeschichten von Johann Matthias Schröckh und Heinrich Philipp Konrad Henke, genannt seien auch Gottlieb Jacob Plancks kritische Arbeiten zur Entwicklung des lutherischen Lehrbegriffs. Marheinekes Reformationsgeschichte ist streng an den Quellen erarbeitet, an Johann Georg Walchs Lutherausgabe, an Veit Ludwig von Seckendorffs historischem Kommentar, Valentin Ernst Löschers Reformations-Acta und Documenta und anderen, und ebenfalls nicht ungelehrt. Um aber ihre Eigenart deutlich zu machen, seien einige Sätze aus dem ersten Kapitel Von der Beschaffenheit der christlichen Kirche zu Anfang des sechszehnten Jahrhunderts und wie es sich allmählich zu einer Reformation angelassen zitiert:

Die Kirche Jesu Christi, von Alters ein reiner Quell der Wahrheit und Seligkeit, in Sonderheit aber in ihrer Jugend blühend in hoher Einfalt des Glaubens und Gottesdienstes, auch mit dem Blute nicht weniger Märtyrer reich geziert, war im Verlauf der Zeiten endlich von ihrer wahren Bestimmung immer weiter abgekommen. [...] Der Mittelpunkt alles Elends in der Kirche war dazumal Rom und dessen päpstlicher Stuhl. [...] Fürwahr die Schuld lag nicht allein an den Päpsten, sondern auch an dem Papstthum. Dasselbe war endlich, da es sein ganzes Wesen entwickelt hatte, zu Anfang des sechszehnten Jahrhunderts so sehr ein Reich von dieser Welt, eitel und irdisch geworden, dass schon ein weltlicher Fürst, Kaiser Maximilianus I. dasselbe zu übernehmen ernsthaft gesonnen war. ${ }^{8}$

So schrieb damals selbstverständlich kein kritisch-pragmatischer Kirchengeschichtler. Marheineke gebraucht vielmehr einen gemütvollen, altväterlichen Stil, der sich an altprotestantische Darstellungen wie Johann Mathesius' Predigten über Luthers Leben oder Friedrich Myconius' Erinnerungen an die Reformation anlehnt. Es kommen auch die Quellen selbst in langen Zitaten und Paraphrasen zur Sprache; so wird Luthers nicht sehr bekannte, aber für die Entwicklung der reformatorischen Ekklesiologie bedeutsame Schrift wider den „hochberühmten Romanisten“ Augustin von Alfeld ${ }^{9}$ über 21 Seiten wiedergegeben. ${ }^{10}$ Marheinekes Absicht ist es, nicht aus der kritischen Distanz mehrerer Jahrhunderte über eine große Zeit zu reden, sondern jene Zeit selbst zum Leben zu erwecken und reden zu lassen und seinen eigenen Text soweit möglich deren Stil anzupassen, so dass ein Gesamtbild aus einem Guss entsteht. Er habe,

7 Marheineke 1818, 1-24 (Anm. 6), wieder abgedruckt in: Veillodter / Schreiber 1819, 114-121 (Anm. 6).

8 Philipp Marheineke, Geschichte der teutschen Reformation, Bd. 1, Berlin 1816, 3. 12.14.

9 WA 6, 285-324.

10 Marheineke 1816, 198-219 (Anm. 8). 
schreibt er in der Vorrede, seine Schreibart „der einfachen, ungeschminkten Weise der Alten näher zu bringen gesucht“. Die neueren Werke der pragmatischen Geschichtsschreibung hätten zur Erklärung der Vorgänge sehr scharfsinnig psychologische Motive und politische Interessen bemüht.

Ich habe an eine andere Zeit gedacht und mich mit einem geringeren, sehr untergeordneten und beschränkten Verdienst begnügt, mich selber so wenig, als möglich, mit meinem Urtheil eingemischt, vielmehr fast durchgängig meine Urkunden und Actenstücke reden lassen. Dieses ist wirklich das beste und einzige Mittel, die Wahrheit und Lauterkeit der Geschichte zu retten und wieder herzustellen, wenn sie genugsam getrübt ist durch Meinungen und Muthmaaßungen, die sich sonst zuletzt gar als Thatsachen gebehrden. ${ }^{11}$

Die zeitgenössischen Rezensenten haben diese Schreibart bemerkt und durchaus anerkennend beurteilt. Die Jenaischen Allgemeinen Literatur-Zeitung bescheinigt eine „feine Schreibart“, die sich „der alterthümlichen genähert hat, ohne doch ins Gezierte oder den Chronikstil zu fallen“, und lobt, dass der Verfasser nicht wie andere protestantische Darsteller seine Unparteilichkeit durch distanzierte oder gar absprechende Urteile über die Reformation habe beweisen wollen. ${ }^{12}$ Die Heidelbergischen Jahrbücher der Litteratur schreiben von einem „musivischen Werk“, d.h. einem Mosaik, dessen Teile ohne großen Aufwand zu einem einzigen, schlichten und ansprechenden Ganzen zusammengekittet seien. ${ }^{13}$ Noch der Erlanger lutherische Dogmatiker Franz von Frank in seiner neueren Theologiegeschichte, nicht unbedingt ein Freund von Marheinekes Theologie, lobte doch, dass sich dessen Reformationsgeschichte durch markige Sprache und Objektivität von der Seichtigkeit pragmatischer Darstellungen abhebe. ${ }^{14}$ Ansonsten galt das Werk noch lange als volkstümlich im besten Sinne des Wortes. ${ }^{15}$ Weniger gelungen fand es, wie zitiert, Schleiermacher, weder die Schreibart noch die Komposition noch auch die Auswahl des Stoffes.

11 Marheineke 1816, XXVII-XXVIII (Anm. 8). Vgl. zum Stil auch ders., Geschichte der teutschen Reformation ${ }^{2}$, Bd. 1, Berlin 1831, XXXIII: „Bei jedem andern geschichtlichen Stoff, an welchem die Form nicht so, wie bei diesem, einen besondern Werth für sich hat, würde ich selbst eine solche Darstellungsweise, wie in sich selbst unausführbar, so auch ganz unangemessen finden. Hier hingegen nimmt, nächst dem Inhalt, auch die Alterthümlichkeit teutscher Denkart und Sprache, der einfache, ungeschmückte Ton jener alten Erzählungen unser Interesse in Anspruch. Es ist die fromme, biedere, treuherzige Weise, zu denken und zu empfinden, wie wir sie sonderlich bei den teutschen Fürsten jener Zeit finden, die auch ihrem Ausdruck ein eigenthümliches, ehrwürdiges Gepräge giebt. Es ist insonderheit die originelle Kern- und Kraftsprache Luthers“.

12 Jenaische Allgemeine Literatur-Zeitung 14 (1817), Bd. 3, Nr. 157, 313-317. Verfasser ist Johann August Martin Haasenritter, ein fleißiger Rezensent, damals Pfarrer in Burgwerben bei Weißenfels.

13 Heidelbergische Jahrbücher der Litteratur 10 (1817), Nr. 54, 849-864.

14 Franz von Frank, Geschichte und Kritik der neueren Theologie der neueren Theologie, insbesondere der systematischen, seit Schleiermacher, hg.v. Richard Grützmacher, Leipzig ${ }^{4} 1908,173$.

15 Karl von Hase, Kirchengeschichte auf der Grundlage akademischer Vorlesungen, Bd. 1, Leipzig 1885, 46; Gustav Wilhelm Frank, Art. „Marheineke“, in: $R E^{3}$, Bd. 12, Leipzig 1903, 304-309, hier 305; Kurt Hünerbein, „Der Berliner Theologe Philipp Konrad Marheineke als Kirchenhistoriker“, JBBKG 54 (1983), 74-96, hier 83-84; Meding 1986, 304 (Anm. 4). 


\section{Schleiermachers Beiträge zum Reformationsjubiläum}

Schleiermachers erwähnte lateinische Festrede hat sich dann offenbar doch bei Marheineke bedient, etwa wenn sie zeigt, dass Luther, wie er selbst später freimütig zugab, zur Zeit des Thesenanschlags noch tief in den päpstlichen Irrtümern und Vorurteilen stak, woraus der Redner folgert, dass der Tag, an dem die Bannandrohungsbulle verbrannt wurde, ein besserer Gedenktag wäre. ${ }^{16}$ Als Reformierter erinnert Schleiermacher daran, dass Luthers Maximen auch diejenigen Zwinglis gewesen seien und dass der alte Zwist beider Parteien nunmehr begraben werde. ${ }^{17}$ Er ruft die Weisheit, Beständigkeit und Großmut der damaligen sächsischen Kurfürsten und die alte deutsche Freiheit ins Gedächtnis, was Gelegenheit gibt zu einem allzu überschwänglichen Lob Friedrich Wilhelms III., der nun - endlich! - die Einrichtung von Presbyterien und Synoden angeordnet habe. ${ }^{18}$ Das reformatorische Prinzip in der Kirche bedeute die immer zunehmende Freiheit in Kirchenordnung und Lehre; auf das akademische Leben angewandt heiße das, dass auch die Praktische Theologie ordentliches Unterrichtsfach sein müsse ${ }^{19}$ und dass insgesamt die Lehr- und Studienfreiheit nicht beschränkt werden dürfe. Dank ihr habe die evangelische Kirche auch scholastischen Dogmatismus, wissenschaftsfeindlichen Biblizismus und Pietismus und westeuropäische Frivolität überstanden. ${ }^{20}$

Außerdem hat Schleiermacher an der Dreifaltigkeitskirche zwei Predigten zum Jubiläum gehalten, eine beim großen Festgottesdienst am Sonnabend, den ersten November, und eine am Sonntag, den zweiten November. Der Festgottesdienst wurde auf königliche Anordnung zusammen mit den Schülern begangen. Der Chor sang Stücke aus dem zweiten Teil des Händelschen Messias über die Boten des Gotteswortes, ${ }^{21}$ und Schleiermacher, der sich als Text Jesu Warnung, einem der Kleinen ein Ärgernis zu geben (Mt 18,5-6), ausgesucht hatte, ging in seiner Predigt besonders auf die Schulkinder und ihre Situation ein: Luther und den anderen teuren Rüstzeugen Gottes sei in summa zweierlei zu verdanken, die Bibel in der Volkssprache und die Lehre von der Gerechtigkeit allein aus Glauben ohne äußere Werke. Die Schulkinder

16 Friedrich Schleiermacher, „Oratio in solemnibus ecclesiae per Lutherum emendatae saecularibus tertiis in Universitate litterarum Berolinensi die III. Novembris A. MDCCCXVII. Habita“, in: Orationes in solemnibus ecclesiae per Lutherum emendatae saecularibus tertiis in Universitate litterarum Berolinensi d. III. Novembr. A. MDCCCXVII. habitae, Berlin o.J. 1817, 14-27, hier 15-16, abgedruckt in: KGA I/10, hg.v. Hans Friedrich Traulsen, Berlin / New York 1990, 1-16, hier 4-5.

17 Schleiermacher 1817, 14-15 (Anm. 16), Schleiermacher 1990, 3-4 (Anm. 16).

18 Schleiermacher 1817, 17-19 (Anm. 16), Schleiermacher 1990, 6-8 (Anm. 16).

19 Schleiermacher 1817, 19-20 (Anm. 16), Schleiermacher 1990, 8-9 (Anm. 16).

20 Schleiermacher 1817, 20 - 26 (Anm. 16), Schleiermacher 1990, 9-13 (Anm. 16).

21 Vgl. Bernhard Schmidt, Lied - Kirchenmusik - Predigt im Festgottesdienst Friedrich Schleiermachers, Berlin / New York 2002, 81-92; Schleiermachers Liedblätter 1817, Berlin / New York 2008, 219236. 
sollten also schon früh in die Lektüre der Heiligen Schrift eingeführt werden, und sie sollten erfahren - was in der Verantwortung der Eltern und Lehrer liege -, dass sie nicht aufgrund von Leistungen und äußerer Folgsamkeit wertgeschätzt und geliebt würden. ${ }^{22}$ (Schleiermachers Adaption der Lehre von der Glaubensgerechtigkeit hat hier gewisse Ähnlichkeit mit derjenigen des Rationalisten Julius Wegscheider: durch Gesinnung statt durch einzelne äußere Werke. ${ }^{23}$ )

Am Sonntag predigte Schleiermacher über Jesu Lobpreis, dass sein Werk den Weisen verborgen und den Unmündigen offenbar sei und dass die Jünger nun sähen, was Könige und Propheten zu sehen begehrt hätten (Lk 10,21-24): Der Gang des göttlichen Wortes und Geistes durch die Geschichte sei beständigen Wechseln unterworfen; auch dem aufscheinenden Licht der Reformation seien wieder Trübungen gefolgt. Christi Verheißung aber stehe, dass sein Werk in allen Kämpfen bleiben und sich durchsetzen werde. ${ }^{24}$

Ein Jahr später, im Herbst 1818, erschien schließlich noch ein durch das Reformationsjubiläum veranlasster Aufsatz Schleiermachers: Der Erfurter Verleger Friedrich Kayser hatte seit Ende 1816 verschiedene Gelehrte um Beiträge zu einem Reformations-Almanach gebeten. ${ }^{25}$ Schleiermachers Beitrag erörtert bleibende Bedeutung und Grenze der protestantischen Bekenntnisschriften; die bleibende Bedeutung sieht Schleiermacher in der für den Protestantismus konstitutiven Abgrenzung gegenüber dem Katholizismus. ${ }^{26}$ Die Frage war inzwischen auch zum Gegenstand einer Kontroverse geworden: Claus Harms, Archidiakon und populärer Prediger an St. Nikolai in Kiel, hatte zum Reformationsjubiläum 95 neue Thesen aufgestellt, die den Rationalismus geißelten und nebenbei auch die Union kritisierten. Der Dresdener Hofprediger Christoph Friedrich Ammon, eigentlich als Rationalist bekannt, nannte Harms' Thesen in seinem Magazin für christliche Prediger eine „bittere Arznei für die Glaubensschwäche der Zeit“ und nahm sie zum Anlass für einen Angriff auf die preußische Union. Schleiermacher beschuldigte Ammon daraufhin in zwei offenen Briefen eines falschen Spiels, während er Harms’ Thesen nur kurz abfertigte, und Harms, der sich jetzt im Kreuzfeuer der Rationalisten wiederfand, war über Schleiermacher, in dem er einen Gleichgesinnten vermutet hatte, ziemlich verbittert. ${ }^{27}$

22 Friedrich Schleiermacher, Am 01.11.1817 vorm. (Samstag) Reformationsjubiläum, Mt 18,5-6*, KGA III/5, hg.v. Katja Kretschmar, Berlin u. a. 2014, 241-258.

23 Julius Wegscheider, Institutiones theologiae Christianae dogmaticae, Halle ${ }^{7} 1833,542-543$ (§ 155). 24 Friedrich Schleiermacher, Am 02.11.1817 vorm. (22. SnT) Lk 10,21-24*, KGA III/5, hg.v. Katja Kretschmar, Berlin u.a. 2014, 260 -267; vgl. Schmidt 2008, 241-244 (Anm. 21).

25 Vgl. Hans-Friedrich Traulsen, Einleitung des Bandherausgebers, KGA I/10, hg.v. Hans Friedrich Traulsen, Berlin / New York 1990, VII-CXVI, hier XXXVII.

26 Friedrich Schleiermacher, „Ueber den eigenthümlichen Werth und das bindende Ansehen der symbolischen Bücher“, in: Reformationsalmanach 2, Erfurt 1819, 335-380, abgedruckt in: KGA I/10, hg.v. Hans Friedrich Traulsen, Berlin / New York 1990, 117-144. Vgl. Martin Ohst, Schleiermacher und die Bekenntnisschriften, Tübingen 1989, 153-157; Gerber 2015, 378- 383 (Anm. 3).

27 Vgl. Hans-Friedrich Traulsen, Schleiermacher und Claus Harms, Berlin-West / New York 1989, 44234. 


\section{Schleiermachers Deutung}

In Schleiermachers Theologie hat die Reformation kategoriale Bedeutung. Das liegt daran, dass die Theologie für ihn nicht die Wissenschaft von Gott oder vom Absoluten ist, sondern immer auf eine geschichtlich gegebene Religionsgemeinschaft und deren Bedürfnisse und Interessen bezogen sein muss. ${ }^{28}$ Die Reformation ist die Epoche der Kirchengeschichte, in der die protestantische Kirche in der Geschichte erschien und ihre Eigenart offenbarte, diejenige Gestalt des Christentums, für die Schleiermacher Theologie treibt. ${ }^{29}$ Insofern geht Schleiermacher etwa in den Einleitungsparagraphen der Glaubenslehre auf den katholisch-protestantischen Gegensatz ein, zu dem sich jede abendländisch-christliche Dogmatik positionieren müsse, ${ }^{30}$ und insofern profiliert er etwa in den Vorlesungen zur Praktischen Theologie zu so ziemlich jedem Thema die katholische und die reformatorische Position gegeneinander. Auch wenn Schleiermacher programmatisch sagen kann, dass die Reformation noch fortgehe ${ }^{31}$ was protestantisch sein will, muss sich auf das Geschehen des 16. Jahrhunderts zurückbeziehen und dort noch mehr auf die Taten und Maximen als auf die Einzellehren der Reformatoren. ${ }^{32}$

Die Reformation sei für Schleiermacher eine Pluralisierung des Christentums, hat man gesagt, der katholisch-protestantische Unterschied Ausdruck zweier individueller Gestaltungen des Christentums, ${ }^{33}$ und das ist auch nicht falsch. Nur: Die sittliche Rechtfertigung der Reformation als reinigendes, kirchenverbesserndes Handeln beruht für ihn gerade darauf, dass die Reformatoren nicht bloß das Christentum um eine

28 Friedrich Schleiermacher, Kurze Darstellung des theologischen Studiums zum Behuf einleitender Vorlesungen, Berlin ${ }^{2} 1830, \S 1-8$, abgedruckt in: KGA I/6, hg.v. Dirk Schmidt, Berlin / New York 1998, 317-446, hier 325-329.

29 Vgl. Gerber 2015, 353-354 (Anm. 3).

30 Friedrich Schleiermacher, Der christliche Glaube nach den Gründsäzen der evangelischen Kirche im Zusammenhange dargestellt ${ }^{2}$, Bd. 1, Berlin 1830, § 23-24, KGA I/13,1, hg.v. Rolf Schäfer, Berlin / New York 2003, 160-169.

31 Friedrich Schleiermacher, Gespräch zweier selbst überlegender evangelischer Christen über die Schrift: Luther in Bezug auf die neue preußische Agende, Leipzig 1827, 85, abgedruckt in: KGA I/9, hg.v. Günther Meckenstock, Berlin / New York 2000, 381-472, 471.

32 Friedrich Schleiermacher, „Die Übergabe des Bekenntnisses als Verantwortung über den Grund der Hoffnung“, in: ders., Predigten. Sechste Sammlung, Berlin 1831, 20 - 24 (abgedruckt in: KGA III/2, hg.v. Günther Meckenstock, Berlin / Boston 2015, 293-303, hier 293-295); „Das Ziel der Wirksamkeit unserer evangelischen Kirche“, in: ders., Predigten. Sechste Sammlung, Berlin 1831, 192-193 (abgedruckt in: KGA III/2, hg.v. Günther Meckenstock, Berlin / Boston 2015, 401-420, hier 401-402); , An die Herren D.D.D. von Cölln und D. Schulz“, in: ThStKr 4, Hamburg 1831, 3-39, hier 7 (abgedruckt in: KGA I/10, hg.v. Hans Friedrich Traulsen, Berlin / New York 1990, 395-426, hier 401); „Vorrede“, in: ders., Predigten. Sechste Sammlung, Berlin 1831, III-XXX, hier IV-VIII (abgedruckt in: KGA III/2, hg.v. Günther Meckenstock, Berlin / Boston 2015, 261-264).

33 Ulrich Barth, „Sichtbare und unsichtbare Kirche“, in: Christentumstheorie. Trutz Rendtorff zum 24.01.2006, hg.v. Klaus Tanner, Leipzig 2008, 179-230, hier 223-224; Constantin Plaul, „Versöhnte Vielfalt“, in: KuD 61 (2015), 248-259, bes. 251-254. 
neue individuelle Ausprägung bereicherten, sondern dass sie bei ihrem Widerspruch gegenüber der damaligen organisierten Kirche gerade die gemeinsame, unhintergehbare Norm für alles Christliche geltend machten, die kanonische Urkunde des Christentums, das Neue Testament. Erst als die organisierte Kirche sich diesen Impulsen verschloss, die Diskussion abbrach und die andere Seite in den Bann tat, begann bei dieser der Aufbau einer eigenen kirchlichen Organisation, die dann, entsprechend der religiösen Eigenart und auch der nationalen und kulturellen Prägung ihrer Träger, auch einen individuellen Charakter annahm. ${ }^{34}$

In der erwähnten Festpredigt bringt Schleiermacher das Programm der Reformation auf zwei Punkte: das Schriftprinzip und die Lehre von der Rechtfertigung allein aus Glauben. Beides - Schleiermacher stellt es so auch sonst zusammen - gehört aber zusammen und lässt zurückführen auf das eine Prinzip: die unmittelbare Gemeinschaft des einzelnen Gläubigen mit dem Erlöser. Einer Kirche als vermittelnder Instanz bedarf es nicht - weder gibt sie die verbindliche Auslegung der Schrift vor, noch legt sie äußere Werke und Handlungen auf, noch auch vermittelt sie in priesterlicher Funktion den Verkehr mit dem Göttlichen. Und insofern ist die Reformation gegenüber dem Katholizismus nicht nur eine Reinigung kraft des Schriftprinzips und eine eigene individuelle Ausprägung des Christlichen, sondern zugleich auch eine spätere Stufe bei der Durchdringung und Formung der Welt durch das christliche Prinzip: In diesem Stadium werden die Laien aus priesterlicher Vormundschaft in die religiöse Mündigkeit entlassen; Geistliche fungieren im Protestantismus nicht mehr als Priester, sondern eher als Lehrer und als Anleiter des gemeinsamen Gottesdienstes. ${ }^{35}$ Die Reformation stellt damit auch ein Stück Heilsgeschichte dar, das Schleiermacher in der anderen genannten Predigt dann mit den Geschicken des göttlichen Wortes in biblischer Zeit parallelisieren kann; sie ist eine Etappe auf dem Weg der Fleischwerdung des göttlichen Geistes, und sie wirkt sich damit auch auf die anderen Gebiete der menschlichen Kultur aus.

\section{Marheinekes Deutung}

Marheinekes Dogmatik geht von einer Wissenschaftslehre nach Schelling und dann nach Hegel aus: Wissenschaft ist das freie Leben des Geistes, und die theologische Dogmatik ist ein System von wissenschaftlichen Sätzen und Erkenntnissen, der Begriff dessen, was in der Religion als Leben erscheint. ${ }^{36}$ Des nach Schleiermacher not-

34 Vgl. Gerber 2015, 136-138.153-157 (Anm. 3)

35 Vgl. Gerber 2015, 162-163.375-376.383-385.392-393.408-410 (Anm. 3)

36 Philipp Marheineke, Die Grundlehren der christlichen Dogmatik, Berlin 1819, 3-6.13-14.16-17.24-25.28-33 (§ 1-6.19-20.23-24.34-35.40-46); ders., Die Grundlehren der christlichen Dogmatik, Berlin ${ }^{2} 1827,3-5$ (§ 1-5). Vgl. Elise Ihle, Philipp Konrad Marheineke. Der Einfluß der Philosophie auf sein theologisches System, Leipzig 1938, 36-50; Eva-Maria Rupprecht, Kritikvergessene Spekulation, Frankfurt am Main 1993, 101-113.123-128.170 -177; Joachim Ringleben, „Philipp Konrad 
wendigen konfessionellen Standpunkts bedarf Marheinekes Dogmatik nicht. Andererseits ist Marheineke aber auch der Vater der neueren Symbolik und Konfessionskunde, ${ }^{37}$ und hier macht er die verschiedenen Objektivierungen oder individuellen Spielarten, in und unter denen das Christentum in der Wirklichkeit erscheint, zum Gegenstand. Marheineke wäre sicher noch besser als Schleiermacher zum geistigen Vater einer Ökumenik als versöhnter Vielfalt der Individualitäten ${ }^{38}$ geeignet. Jede christliche Religionspartei sammle sich um eine religiöse Hauptidee und schaffe sich ein Glaubensbekenntnis oder Symbol, um diese Idee $\mathrm{zu}$ formulieren, um sich der eigenen Identität gewiss zu werden und um diese nach außen zu profilieren; so seien also die Symbole die Form, die die ewige christliche Wahrheit in der Zeitlichkeit annehme. ${ }^{39}$ Bedeutsam ist nun, dass Marheineke die Symbole und die sich in ihnen konzentrierenden Individuationen des Christentums in ein genaues Verhältnis zu Nation und Staat setzt: In den Symbolen spreche sich auch der Nationalcharakter aus, und die Formulierung und Verkündung eines Symbols geschehe unter Approbation des Staates. ${ }^{40}$ In 1814 anonym veröffentlichten Aphorismen zur Erneuerung des kirchlichen Lebens schreibt Marheineke gar, ein Volk habe seine nationale Eigenart, Sitte und Sprache allein in der Religion, und so seien Kämpfe um das Christentum innerhalb eines Volks zugleich Kämpfe um den Nationalcharakter. Dieser äußere und vollende sich im formulierten Symbol. Auch der Staat hänge daran, und somit seien die Symbole immer auch rechtlich verpflichtend. ${ }^{41}$

Seine Reformationsgeschichte hat Marheineke bewusst als Geschichte der ,teutschen“ Reformation geschrieben. Ihr Held ist weniger Luther als Deutschland; ihr Leitmotiv ist es, dass Deutschland die ihm von Rom auferlegte Gestalt des Christentums überwindet und zu seiner eigenen, modern gesprochen „kontextuellen“ Frömmigkeit und Theologie findet; ${ }^{42}$ damit einher geht der Kampf auch um die politische Freiheit. ${ }^{43}$ (Völlig fremd sei leider dem jungen Kaiser das deutsche Wesen geblieben,

Marheineke (1780-1846) - die Zuflucht im Begriff“, in: Stiftsgeschichte(n), hg.v. Bernd Schröder / Heiko Wojtkowiak, Göttingen 2015, 59-65.

37 Vgl. Ferdinand Kattenbusch, Confessionskunde, Bd. 1, Tübingen 1892, 52-56; Hünerbein 1983, 75 - 78 (Anm. 15); Simon Gerber, „Schleiermacher und die Kirchenkunde des 19. Jahrhunderts“, ZNThG 11 (2004), 183-214, hier 201-203.

38 Vgl. Plaul 2015, 250-251.254-259 (Anm. 33).

39 Philipp Marheineke, Christliche Symbolik oder historischkritische und dogmatischkomparative Darstellung des katholischen, lutherischen, reformirten und socinianischen Lehrbegriffs; nebst einem Abriß der Lehre und Verfassung der übrigen occidentalischen Religionspartheyen, wie auch der griechischen Kirche, Bd. 1,1, Heidelberg 1810, 5-10.

40 Marheineke 1810, 15-17.29-31 (Anm. 39).

41 [Philipp Marheineke], Aphorismen zur Erneuerung des kirchlichen Lebens im protestantischen Deutschland, Berlin 1814, 9-23.32-36.

42 Marheineke 1816, 15.27.137-138.140.154.162.183.353 (Anm. 8); ders., Geschichte der teutschen Reformation, Bd. 2, Berlin 1816, $160-161$.

43 Marheineke 1816, 51-52.83.108.119.151.196-198.248-250.275-276 (Anm. 8). Vgl. Winckler 1969, 56-58 (Anm. 4). - Auch in der Reformationspredigt von 1814 (Text: Apg 5,38-39) betont Marheineke die nationale Bedeutung der Reformation, vgl. Marheineke 1818, 55 - 76 (Anm. 6). 
ein Grund dafür, dass halb Deutschland katholisch blieb. ${ }^{44}$ ) Die Reformation ist überhaupt ein Gemälde alter deutscher Freiheit, Sitte und Gesinnung und auch Kunst. ${ }^{45}$

Wie die Reformation zu ihrer Zeit eine wahre Nationalangelegenheit war, für welche sich jeder interessirte, mochte er ihr zugethan seyn oder nicht, so dachte ich mir immer, müßte sich auch ihr Geist und Wesen in der Geschichte auffassen und darstellen lassen: denn wie überhaupt das religiöse Gefühl des Herzens sich nach und nach zur klaren Einsicht und Erkenntniß des Verstandes erhebt, so stieg auch hier, so recht aus dem Herzem des teutschen Volks, dieser sich immer mehr reinigende Glaube und die so rührend fromme Theilnahme daran allmählig bis zu den Häuptern der Nation empor. [...] Den Kern der teutschen Geschichte, die Blüthenzeit des christlichen Glaubens teutscher Nation, stellet die Reformation uns dar. ${ }^{46}$

Schleiermacher wie Marheineke sehen in der Reformation sowohl ein individuelles als auch ein für das ganze Christentum Geltung beanspruchendes Moment; aber für Schleiermacher ist der Impuls auf das gesamte Christentum das Ursprüngliche, der auch an mehreren Orten gleichzeitig und unabhängig voneinander ausgeübt worden sei, in Sachsen, der Schweiz und Frankreich. ${ }^{47}$ Für Marheineke ist die Reformation umgekehrt zunächst eine auch kulturell bedingte neue Individuation des Christentums, die sich dann zu allgemeiner Bedeutung aufschwingt.

Krönender Abschluss von Marheinekes Werk ist die Verlesung der Augsburger Konfession, durch die das Errungene als öffentliches Symbol und als eigene Gestalt der ewigen, allgemeinen christlichen Wahrheit zur Erscheinung kommt.

Es war einer der schönsten Tage der Christenheit. [...] Ein neues Gefühl durchlebte und durchdrang sie von diesem großen Augenblick an. Durch das feste Band eines gemeinsamen Glaubens fühlten sie sich jetzt mehr denn je zuvor innig verbunden. [...] Vor Kaiser und Reich, ja vor der ganzen christlichen Welt standen sie, mit einem großen Gebet im Herzen, ihre Rechtfertigung darstellend in ihrem Bekenntniß, in vollkommenster Einigkeit mit allen wahrhaft gläubigen und christlichen Gemüthern in der ganzen Welt und auf einer Höhe, von wo sie mit göttlicher Zuversicht auf viele Jahrhunderte hinsehen konnten. ${ }^{48}$

44 Marheineke 1816, XII. 272 (Anm. 8).

45 Marheineke 1816, 83.151.154.254.268 (Anm. 8).

46 Marheineke 1816, XXIV-XXV (Anm. 8).

47 Friedrich Schleiermacher, Vorlesungen über die Kirchengeschichte, Kolleg 1821/22 Nachschrift Klamroth, 92.-93. Stunde, KGA II/6, hg.v. Simon Gerber, Berlin / New York 2006, 629-630. - Schleiermacher kann den Protestantismus einmal als die den germanischen Völkern angemessene Form des Christentums den romanischen Katholizismus gegenüberstellen (Christliche Sitte 1822/23, Sämmtliche Werke I/12, Berlin 1843, 139); aber das ist erst eine sekundäre Erscheinung. Die reformatorische Idee kommt aus dem Christentum selbst, nicht aus der individuellen Beschaffenheit ihrer Protagonisten. Vgl. Gerber 2015, 145-149.357-358 (Anm. 3).

48 Marheineke 1816, 458 - 460 (Anm. 42). 


\section{Blick in die Zukunft und Gegenwart}

Als Schleiermacher kurz nach dem Jubiläum in der Vorlesung über die Praktische Theologie darauf zurückblickte, meinte er, es sei in seiner Feier bestimmter Personen (gemeint wird besonders Luther sein) geradezu katholisch gewesen. ${ }^{49}$ In seiner Festpredigt hat er selbst die „theuren Rüstzeuge des Herrn“ und deren „große kräftige Zeit mit ihren Anstrengungen und Kämpfen“ gepriesen, ${ }^{50}$ will das reformatorische Prinzip aber ansonsten vor allem für Gegenwart und Zukunft geltend machen, auch über das im 16. Jahrhundert Erreichte hinaus. Das bedeute insbesondere die Überwindung des seinerzeit wohl notwendigen, inzwischen aber obsoleten landeskirchlichen Kirchenregiments, aber auch die freisinnige Weiterentwicklung des Lehrbegriffs auf Grundlage des Schriftprinzips. ${ }^{51}$

Marheineke schrieb in der Vorrede seiner Reformationsgeschichte, es sei generell schwierig, das bloß Zeitbedingte und Vorübergehende vom Feststehenden und Bleibenden zu unterscheiden. ${ }^{52}$ Trotzdem wagte er eine Prophezeiung: Die vom Westfälischen Frieden festgeschriebene Bikonfessionalität Deutschlands gehe zu Ende, die öffentlichen Verhältnisse der Kirchen lösten sich auf, noch in diesem Jahrhundert werde ein neuer Luther aufstehen, und die vom ersten Luther so nicht beabsichtigte schmerzliche Trennung werde überwunden werden. „Denn was die Menschen trennet, ist allein das Menschliche, das Göttliche vereiniget sie immerdar.“53

Einig waren sich Marheineke und Schleiermacher darin, dass die lutherisch-reformierte Union die Sache der Reformation legitim weiterführe. Am Palmsonntag 1822 führten beide als Pfarrer an ihrer Dreifaltigkeitsgemeinde zusammen die Union ein. ${ }^{54}$ Wenige Jahre später - Marheineke stand inzwischen stärker unter dem Eindruck der Hegelschen Philosophie - stießen dann beider Reformationsdeutungen hart zusammen. Schleiermacher bestritt unter dem Pseudonym „Pacificus Sincerus“ das von Friedrich Wilhelm III. beanspruchte Recht des Landesherrn, seiner Landeskirche als summus episcopus eine Agende zu verordnen: Geltendes Recht, ältere Kirchengeschichte und Reformation kennten keine solche Kompetenz, sondern nur ein obrigkeitliches Aufsichtsrecht circa sacra, ${ }^{55}$ und auch die noch verbreitete Konsistorial-

49 Friedrich Schleiermacher, Vorlesungen zur Praktischen Theologie 1817/18, Nachschrift Jonas, Schleiermacher-Nachlass 550, 54v (erscheint in: KGA II/11).

50 Schleiermacher 2014, 242 (Anm. 22).

51 Vgl. Gerber 2015, 374-378 (Anm. 3).

52 Marheineke 1816, V-IX (Anm. 8).

53 Marheineke 1816, XIV-XXIV (Anm. 8).

54 Vgl. Andreas Reich: Friedrich Schleiermacher als Pfarrer, Berlin / New York 1992, 146-170; Schmidt 2002, 286-318 (Anm. 21).

55 Pacificus Sincerus [Friedrich Schleiermacher], Ueber das liturgische Recht evangelischer Landesfürsten, Göttingen 1824, 3-67, abgedruckt in: KGA I/9, hg.v. Günther Meckenstock, Berlin / New York 2000, 213-256. 
verfassung müsse im Namen der Reformation überwunden werden. ${ }^{56}$ Marheinekes Gegenschrift beklagte den schmerzlichen Mangel an einem nationalen evangelischen Kirchenrecht, zeitige der doch zu aller Schaden Willkür und Subjektivismus. ${ }^{57}$ Die evangelische Kirche lebe weder als Sekte außer dem Staat noch wie die römisch-katholische Kirche als eigener Staat im Staat, sondern in Harmonie mit ihm und so, dass jede Seite erst durch die jeweils andere das wahrhaft sei, was sie im Geiste des Evangeliums sein solle. Ein evangelischer Staat gestalte sich anders als ein katholischer, und umgekehrt lebe die evangelische Kirche in einem monarchischen evangelischen Staat anders als in einem republikanischen; in jenem repräsentiere eben der Monarch in seiner Person die Einheit von Staat und Kirche. Pacificus Sincerus aber (Marheineke wusste zweifellos, dass sich dahinter Kollege Schleiermacher verbarg) habe für die evangelische Kirche eine ursprüngliche Unabhängigkeit von der Landeshoheit erdichtet, die es so nie gegeben habe; die evangelischen Verfechter einer vom Staat unabhängigen Kirche lebten selbst noch mehr im römischen Katholizismus und hätten das Prinzip der Reformation verkannt. ${ }^{58}$ Mit anderen Worten: Staatskirche und landesherrliches Kirchenregiment waren kein Notbehelf, sondern bleiben ureigenster Ausdruck eines im reformatorischen Sinne organisierten Gemeinwesens.

Als 1831 eine zweite Auflage der Reformationsgeschichte erschien, erweitert um zwei weitere Bände über die Zeit von der Übergabe der Augsburger Konfession bis zum Religionsfrieden, schrieb Marheineke zum Geleit - es war das Jahr nach der Julirevolution -, diejenigen Völker, die sich damals dem römischen Stuhl entzogen hätten, hätten mit der gereinigten Lehre auch Mündigkeit des Geistes, Gedanken- und Gewissenfreiheit und eine in diesem Sinne geordnete Staatsverfassung erlangt.

Im Besitz dieser kirchlichen und politischen Freiheit, dürfen sie nicht, wie so viele andere Völker, welche jetzt dem römischen Stuhl widerstreben, erst darnach ringen und durch die Schule blutiger Revolutionen und namenloser Leiden gehen, sondern nur treu bewahren und sorgsam pflegen und ausbilden, was sie bereits haben und ihnen durch die treuen Bemühungen ihrer Vorfahren, christlicher Lehrer und Fürsten im schönsten Einklang [...] erworben worden ist. ${ }^{59}$

56 Pacificus Sincerus [Schleiermacher] 1824, 67-90 (Anm. 55), Schleiermacher 2000, 256-269 (Anm. 55).

57 Philipp Marheineke, Ueber die wahre Stelle des liturgischen Rechts im evangelischen Kirchenregiment, Berlin 1825, 1-2.

58 Marheineke 1825, 3-26. 31-53. 71-72. 88 (Anm. 57). Vgl. Markeineke 1827, 335-336 (§ 525-526) (Anm. 36).

59 Marheineke 1831, XXXIV-XXXV (Anm. 10). 


\section{Zeittafel}

1805 Beginn des Briefkontakts zwischen Philipp Marheineke (Erlangen) und Friedrich Schleiermacher (Halle)

1806 Schließung der Universität Halle durch Napoleon

1807 Marheineke folgt einem Ruf an die Universität Heidelberg.

1809 Schleiermacher wird reformierter Prediger an der Berliner Dreifaltigkeitsgemeinde.

1810 Schleiermacher wird Professor an der neuen Berliner Universität.

1810-13 Marheineke, Christliche Symbolik

1811 Marheineke folgt einem durch Schleiermacher übermittelten Ruf an die Universität Berlin.

1814 [Marheineke,] Aphorismen zur Erneuerung des kirchlichen Lebens im protestantischen Deutschland

1816 Marheineke, Geschichte der teutschen Reformation, Bd. 1-2

1817 Marheineke, Predigt zum Reformationsjubiläum in Hanau (14.9., Text: Mt $8,23-27)$

Aufruf Friedrich Wilhelms III. zur Union (27.9.)

Gemeinsame Abendmahlsfeier der lutherischen und reformierten Mitglieder der Berliner Synode (30.10.)

Schleiermacher, Festpredigt zum Reformationsjubiläum in der Dreifaltigkeitskirche (1.11., Text: Mt 18,5-6)

Schleiermacher, Predigt zum Reformationsjubiläum in der Dreifaltigkeitskirche (2.11., Text: Lk 10,21-24)

Schleiermacher, Lateinische Festrede zum Reformationsjubiläum an der Berliner Universität (3.11.)

1818 Marheineke, Fünf Reformations-Predigten (darunter eine für 1817 über Joh $8,31-32)$

Schleiermacher, „Ueber den eigenthümlichen Werth und das bindende Ansehen symbolischer Bücher“

1820 Marheineke erhält die erste (lutherische) Pfarrstelle an der Dreifaltigkeitsgemeinde

1822 Einführung der Union an der Berliner Dreifaltigkeitsgemeinde

1824 Pacificus Sincerus [Schleiermacher], Ueber das liturgische Recht evangelischer Landesfürsten

1825 Marheineke, Ueber die wahre Stelle des liturgischen Rechts im evangelischen Kirchenregiment

1831 - 34 Marheineke, Geschichte der teutschen Reformation ${ }^{2}, B d .1-4$ 
